\title{
ACTIVE VIBRATION CONTROL PROTOTYPING IN ANSYS: A VERIFICATION EXPERIMENT
}

\author{
Ing. Gergely TAKÁCS, PhD.* \\ * Institute of Automation, Measurement and Applied Informatics \\ Faculty of Mechanical Engineering \\ Slovak University of Technology in Bratislava \\ Námestie Slobody 1, 81231 Bratislava 1 \\ gergely.takacs@stuba.sk
}

\begin{abstract}
The paper demonstrates the possibility to use finite element analysis packages for the prototyping of control algorithms for active vibration control. A model of a laboratory demonstration device consisting of a clamped beam with piezoceramic actuators has been prepared in ANSYS. The free response of this system is compared to laboratory measurements to verify the validity of the base model. A simple linear quadratic (LQ) control system and a state observer have been implemented into the native parametric design language of ANSYS. The complex electro-mechanical transient response of the control system has been compared to laboratory measurements, verifying the validity of the proposed approach.
\end{abstract}

Keywords: vibration control, finite element analysis, control system prototyping, linear quadratic control

\section{INTRODUCTION}

Vibration, or in other words mechanical oscillations around an equilibrium point, may be both useful but can unfortunately be extremely harmful as well. Undesired vibration levels may affect product lifecycle, decrease product quality or cause economic loss. In some cases, unwanted mechanical vibration may even threaten human life and health. Passive approaches to damp vibrations have been at the hands of engineers for a long time. These methods usually involve a form of insulation or change in mass, stiffness or geometrical properties of the system in question. While passive methods certainly do have their merits and are effective for higher bandwidths, in certain situations the use of semi-active or active vibration control methods is necessary $[1,5,6,7,8]$.

Active vibration control is a method, where the undesired vibration in a mechanical structure is damped using actuators, which gain their feedback based on a control algorithm making use of real-time sensor measurements [2,3,8,9]. While the design of such control systems and algorithms traditionally has been the role of control engineers, the nature of the vibration engineering task, requires a good understanding of the underlying mechanical properties of the structure and the vibration dynamics phenomena itself.

The advent of finite element analysis and modeling (FEA,FEM) software in the past two decades made the performance of dynamic vibration analysis possible; including modal analysis, harmonic analysis and the numerical modeling of transient effects. Although these tools allow engineers to perform a detailed analysis of the vibration properties of mechanical systems, they traditionally do not implement any means to test and implement an active control system. 
The aim of this article is to demonstrate the hidden possibilities given by the ANSYS finite element software in the field of control system prototyping for active vibration attenuation systems. While normally ANSYS does not have the tools and means to include the effect of control systems in dynamic simulations, it is possible to use the ANSYS Parametric Design Language (APDL) to include digital control systems in a transient analysis. An engineer faced with a finished product certainly will not perform lengthy and complicated transient analyses in ANSYS, instead they will use the usual "tools of the trade" for control engineering. However, in the design and prototyping stages it can be extremely important to include the dynamic effects of a control system.

It has already been demonstrated that it is possible to program a simple proportional integrating derivative (PID) controller using the APDL programming language of ANSYS and perform a controlled transient analysis [4]. The current paper models a demonstration structure with active vibration control in ANSYS, including a linear quadratic (LQ) control algorithm and a basic state observer. The simulation data obtained via the ANSYS simulation will be compared to laboratory measurements to verify the validity of the approach.

\section{THE VIBRATION CONTROL SYSTEM}

To demonstrate the potential of control system prototyping in ANSYS, a simple laboratory demonstration hardware has been used as a benchmark example. This device consists of a clamped aluminum beam with the dimensions $550 \times 40 \times 3 \mathrm{~mm}$, equipped with MIDÉ QP 16n piezolectric patches [1]. These transducers are mounted in multiple locations; the ones closer to the clamped end are used as actuators to the control system while the rest are close circuited in order to prevent significant interaction with the electro-mechanical properties of the system. The feedback to the control algorithm is provided by an industrial laser triangulation system, taking direct measurements of the deflection at the free end. The scheme of the full device is illustrated in Fig. 1. The capacitive sensor, electrodynamic shaker and the piezoceramic patch PZT3 have not been used in this experiment. The control system and measurement have been performed on the Matlab xPC Target rapid software prototyping system using a host and a target controller computer. 


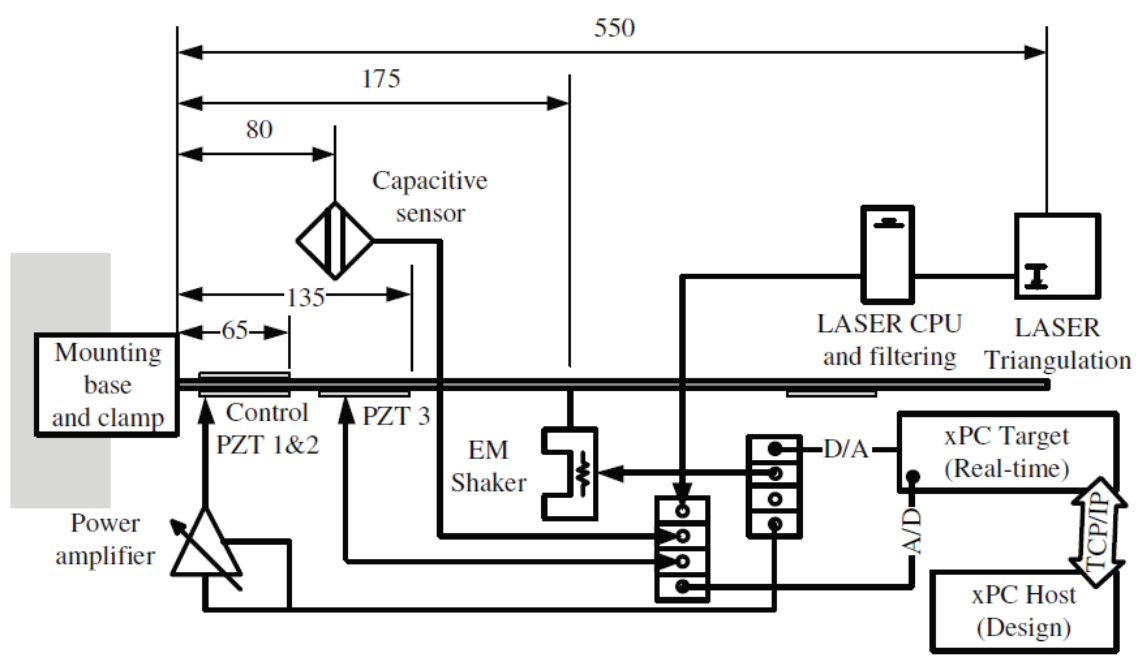

Fig. 1 - Functional scheme of the demonstration device

\section{MODELING THE FREE RESPONSE OF THE VIBRATION CONTROL SYSTEM IN ANSYS}

The geometric model prepared in the ANSYS finite element analysis package mirrors the dimensions of the real device. The piezoelectric patches placed in their respective locations have also used the dimensions of the ceramic patches mounted on the physical device. The solid model did not utilize a clamp structure; instead, it assumed a perfect clamp at the base of the beam entering the structure.

Material properties of the beam and the piezoelectric patches have been determined according to manufacturer's specifications and commonly available engineering data. Both the beam and piezoelectric patch were meshed using the SOLID5 element, which provides an electromechanical coupling modeling the piezoelectric element [11]. Loadings have been specified at the base of the beam, restricting nodal displacement in all directions, defining a nodal force at the end of the beam modeling the manual displacement of the end, gravity acceleration component and electrical properties of the piezoceramics. The piezoelectric transducers have a ground terminal defined, while the other terminal was supplied with a constant $0 \mathrm{~V}$ potential in the free tests [11]. The meshed model of the smart beam is illustrated in Fig. 2.

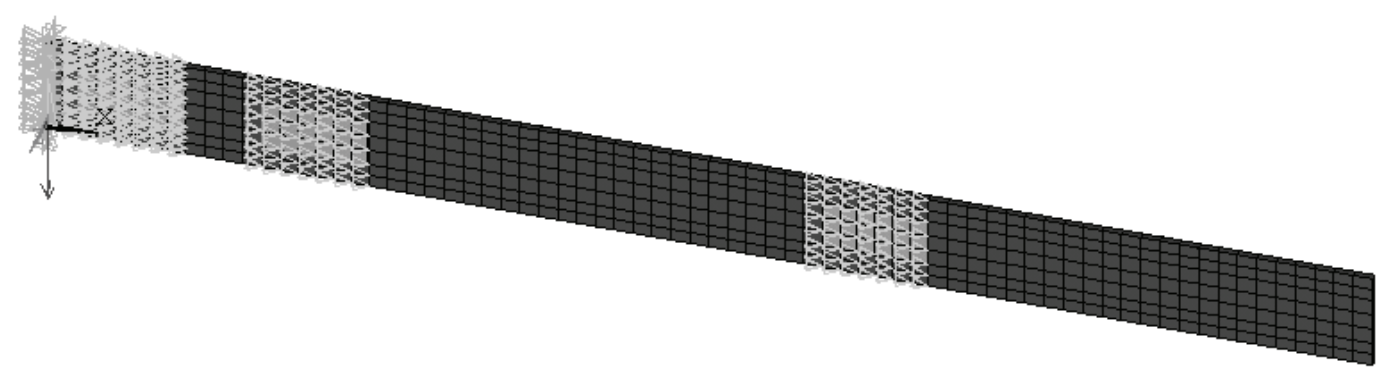


Fig. 2 - Meshed model of the composite smart device

The model is then loaded at the end with a nominal force impulse of $2.4 \mathrm{~N}$, modeling the effect of deflecting the free end of the beam to approximately $10 \mathrm{~mm}$ away from the equilibrium position and letting it go without any further force interactions. The long beam with a small stiffness is a very flexible structure and settling time ranges in the area of several seconds up to even one minute. The damping properties of the structure are very important in obtaining a proper transient response. As damping phenomena are generally hard to model in finite element packages, the simple proportional damping model has been chosen, given by $[3,11]$ :

$$
C=\alpha M+\beta K
$$

where $C$ is the resulting damping matrix, $M$ is the mass matrix, $K$ the stiffness matrix; while $\alpha$ is the mass damping coefficient and $\beta$ is the stiffness damping coefficient. For the structure in question a coefficient of $\alpha=0$ and $\beta=0.00015$ has been chosen. The very small coefficient is due to the flexible nature of the beam. As the experimental tests will demonstrate it is a valid and realistic decision.

The flexible nature of the mechanical system also has an effect on the dynamic behavior of the beam. The response is dominated largely by the first mode of vibration, located at approximately $8.2 \mathrm{~Hz}$ according to the modal analysis results. Previous experiments on the beam also indicate that this is a valid approximation of the system dynamics [1]. The fact that the beam dynamics are largely concentrated within this bandwidth has further implications on control system design. The real-time application of modern optimization and model-based algorithms still requires significant simplifications of the dynamics models [1]. Therefore, only the dynamics associated with the first mode have been reviewed here, setting the simulation time steps at 0.01 seconds. According to the usual rule of thumb in ANSYS, the $100 \mathrm{~Hz}$ bandwidth being more than 20 times higher than the largest mode of interest, is a sufficiently large resolution.

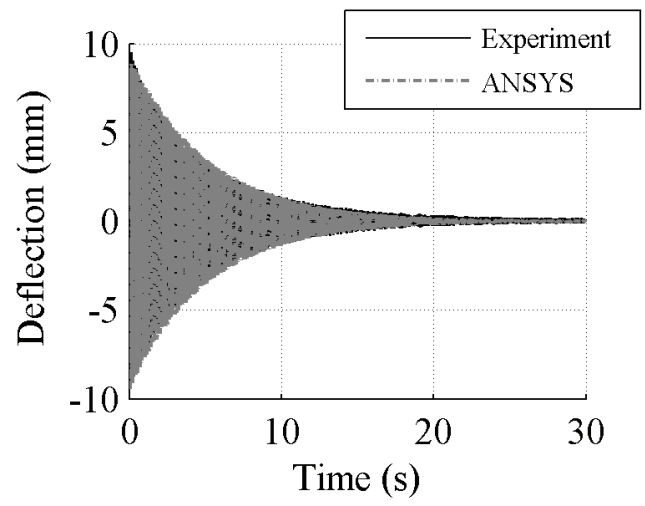

(a) Full response from 0 to 30 seconds

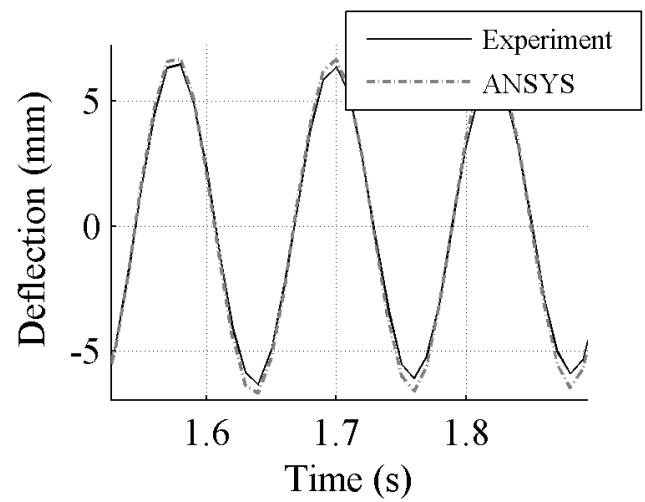

(b) Detailed section of the response

Fig. 3 - Free transient response of the smart beam to an initial force effect displacing the tip approximately $10 \mathrm{~mm}$ away from the equilibrium position 
The free response of the beam has been therefore simulated in the time range of 0 to 30 seconds with a 0.01 second resolution. The initial displacement of the beam has been simulated using a $2.4 \mathrm{~N}$ force impulse lasting 0.01 seconds at the beginning of the simulation (Not shown.). The free response of the beam is illustrated in Fig. 3 where (a) shows the full response and (b) a detail of the transient behavior. As it is clearly visible from both images, the free response of the model to a force effect displacing the tip approximately $10 \mathrm{~mm}$ away from the equilibrium position conforms to the laboratory measurements. The simulated and real response of the electromechanical behavior of the structure without control is essentially the same.

\section{MODELING THE CONTROLLED RESPONSE OF THE VIBRATION CONTROL SYSTEM IN ANSYS}

The control system considered for the actively controlled beam structure is linear quadratic (LQ). Let us model the dynamics of the beam with a simple second order state-space equation given by $[1,3,10,8,9]$ :

$$
\begin{aligned}
& x_{k+1}=A x_{k}+B u_{k} \\
& y_{k}=C x_{k}
\end{aligned}
$$

where the state, input and output matrices $A, B$ and $C$ are determined based on the dynamics of the modeled system, without assuming the presence of direct output feedback. Let us now assume a fixed matrix feedback $K$, which determines the inputs to this system based on state observations given by $[1,2,3]$ :

$$
u_{k}=-K x_{k}
$$

This fixed feedback $K$ must minimize the linear quadratic cost function given as [2,3]:

$$
J=\sum_{k=0}^{\infty}\left(x_{k}^{T} Q x_{k}+u_{k}^{T} R u_{k}\right)
$$

using the state penalty chosen as $Q=C^{T} C \quad(-)$ and the low input penalty chosen to be $R=1 E-4(-)$. The LQ feedback for the given state-space model of the composite beam dynamics has been calculated using Matlab, and the state observer gain $y_{k}$ has been similarly determined by the aid of this environment. This discrete control system has been then implemented into the ANSYS parametric design language, using a controller cycle. Essentially, after each iteration of the transient simulation (divided by steps of 0.01 seconds) a measurement of the tip deflection has been taken. Based on the actual tip deflection the state of the system has been approximated to get $\breve{x}$, which then allows the computation of the control input to the piezoelectric transducers. The input to the transducers has been saturated by $120 \mathrm{~V}$ to prevent the ceramics from depolarization.

Figure 4. shows the results of the simulation of the structure under LQ control and its comparison to the real laboratory measurements. The figures not only demonstrates that the vibration settling time has been reduced from 30 seconds to well under 3 seconds, but also shows an agreement of 
the modified ANSYS code and experimental results. Figure 4. (a) illustrates the transient response of the beam under linear quadratic control in response to an initial displacement of $10 \mathrm{~mm}$. The simulated response coincides with the laboratory measurement results. The voltage supplied to the piezoelectric transducers in response to the displacements is shown in Fig. 4. (b).

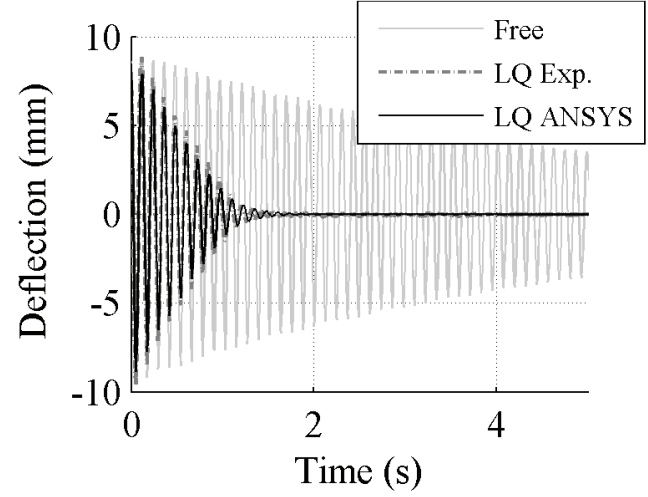

(a) Beam tip displacement under control

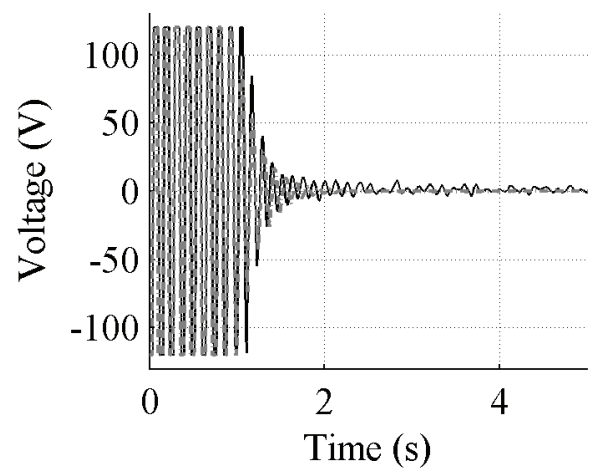

(b) Voltage supplied to the amplifiers

Fig. 4 - Transient response of the smart beam under linear quadratic control to an initial force effect displacing the tip approximately $10 \mathrm{~mm}$ away from the equilibrium position

The initial phase of the control course shows a very saturated "bang-bang" control, which is a logical result of the physical limits of static mode piezoceramics. The experimental system shows small displacements after the 1.5 second mark, which is a result of additional disturbances and mechanical noise present in the laboratory. This noise has not been modeled in the FEA analysis, therefore their effects are not shown in the results.

\section{CONCLUSIONS}

Finite element packages such as ANSYS are valuable in the design of control systems for structures and products featuring active vibration attenuation. As the results presented in this paper demonstrate, the proposed method produced simulation results which coincide with laboratory measurements.

The author would like to thank the financial support granted by the Slovak Research and Development Agency under the contracts APVV 0910-10 and APVV 0131-10.

\section{REFERENCES}

[1] TAKÁCS, G - ROHAL'-ILKIV, B. Predictive Vibration Control: Efficient MPC of Lightly Damped Beams. Springer-Verlag, London, United Kingdom. To be released in 2012. First edition.

[2] FUlleR C. R., ELliOT S. J., Nelson P. A. Active Control of Vibration. Academic Press, 1996.

[3] INMAN, D. J. Vibration with control. John Wiley and Sons, Chichester, England. 2006. 
[4] TAKÁCS, G.: Temperature Controlled Welding Simulation : Finite Element Analysis of a PID Controlled Temperature Constrained Arc Welding Process. Saarbrücken, VDM Verlag, 2010.

[5] BEARDS, C.F. Structural Vibration: Analysis and Damping. Arnold, London, United Kingdom. 1996. First edition.

[6] de SILVA, CW.: Vibration: Fundamentals and Practice. CRC Press, Boca Raton, Florida, USA. 2006. Second edition.

[7] INMAN, D.J. Engineering Vibrations. Pearson International Education (Prentice Hall), Upper Saddle River, NJ, USA. 2007. Third edition.

[8] PREUMONT, A.: Vibration Control of Active Structures. Kluwer Academic Publishers. 2002. Second edition.

[9] HATCH, M.: Vibration Simulation Using Matlab and ANSYS. Chapman and Hall / CRC, Boca Raton, Florida, USA. 2000. First edition.

[10] LJUNG, L. System Identification: Theory for the User. PTR Prentice Hall, Upper Saddle River, NJ, USA. 1999. Second edition.

[11] ANSYS, Inc. Help System. Release 13.0 